\title{
Intraoperative inadvertent piercing of the aortic arch: case report
}

\author{
Hye Seon Kim, MBBS, ${ }^{1}$ Dong Hwan Kim, MD, ${ }^{1}$ Kyung Hwan Kim, MD, PhD, ${ }^{2}$ \\ Youn Joung Cho, MD, PhD, ${ }^{3}$ and Chun Kee Chung, MD, $\mathrm{PhD}^{1}$
}

\begin{abstract}
Departments of ${ }^{1}$ Neurosurgery, ${ }^{2}$ Thoracic and Cardiovascular Surgery, and ${ }^{3}$ Anesthesiology and Pain Medicine, Seoul National University College of Medicine and Seoul National University Hospital, Seoul, Korea
\end{abstract}

\begin{abstract}
Aortic injury is a rarely encountered complication of spinal surgery. The authors report a case of a 32-year-old woman with a T3 tumor who experienced an intraoperative aortic arch injury during T3 spondylectomy. The patient was successfully treated with no postoperative neurological deficits.
\end{abstract}

https://thejns.org/doi/abs/10.3171/2020.1.SPINE191156

KEYWORDS spine surgery; vascular injury; vascular complication; thoracic

A ORTIC injury is a rare, but well-recognized complication of spinal surgery. ${ }^{49}$ Even a small injury can cause massive bleeding, leading to tragic outcomes. A thorough understanding of anatomy and planning of the surgery is mandatory to avoid the injury, but unfortunately if it occurs, prompt decision-making is required to control the damage. In this paper we report a case of a 32-year-old woman who experienced an intraoperative aortic injury that was recognized and managed appropriately, leaving no neurological sequelae.

\section{Case Report}

History and Examination

A 32-year-old woman was referred to our tertiary care center (Seoul National University Hospital) with an enlarging tumor in the T3 thoracic spine. A year prior she had undergone resection of the mass at the local hospital due to weakness in her right leg (Fig. 1A and B). Partial removal of the tumor was achieved and the pathological examination revealed a diagnosis of chordoid meningioma. The immediate postoperative MRI showed the remaining mass in the T3 vertebral body, but the epidural mass, which was believed to be responsible for the symptom, had been removed (Fig. 1C and D). After the initial surgery, the weakness improved and she became asymptomatic.
The follow-up MRI was performed 1 year later and showed an increasing size of the tumor, involving the vertebral body and right pedicle and lamina of T3 (Fig. 1E and F). When she visited our center, she had developed paresthesia of her right leg, so we decided to perform a corpectomy of the T3 vertebra based on our impression that the tumor originated from the bone.

\section{Operation and Postoperative Course}

We planned to dissect the aortic arch from the vertebral bodies and ligate the segmental arteries from the aorta prior to the corpectomy, because the aortic arch was adjacent to the T3 vertebral body. With the help of a thoracic surgeon, the dissection and ligation were completed successfully through a thoracoscopy. In addition, the anterior longitudinal ligament (ALL) and annulus fibrosus were partially resected at the T2-3 and T3-4 levels.

After the lateral approach, the patient was turned prone and operated on via the posterior approach. The previously operated laminae of T3 were lifted, followed by resection of bilateral pedicles and ligation of bilateral T3 roots. An incision was then made on the posterior longitudinal ligament and the posterior annulus fibrosus. A small osteotome was used for deepening the annulus fibrous incision at the level of T3-4, when it slipped anteriorly. Immediately, a sudden gush of blood filled the surgical field. An

ABBREVIATIONS ALL = anterior longitudinal ligament; $\mathrm{CPB}=$ cardiopulmonary bypass; $\mathrm{DHCA}=$ deep hypothermic circulatory arrest; $\mathrm{LV}=$ left ventricle; $\mathrm{RCP}=$ retrograde cerebral perfusion; SVC = superior vena cava. 

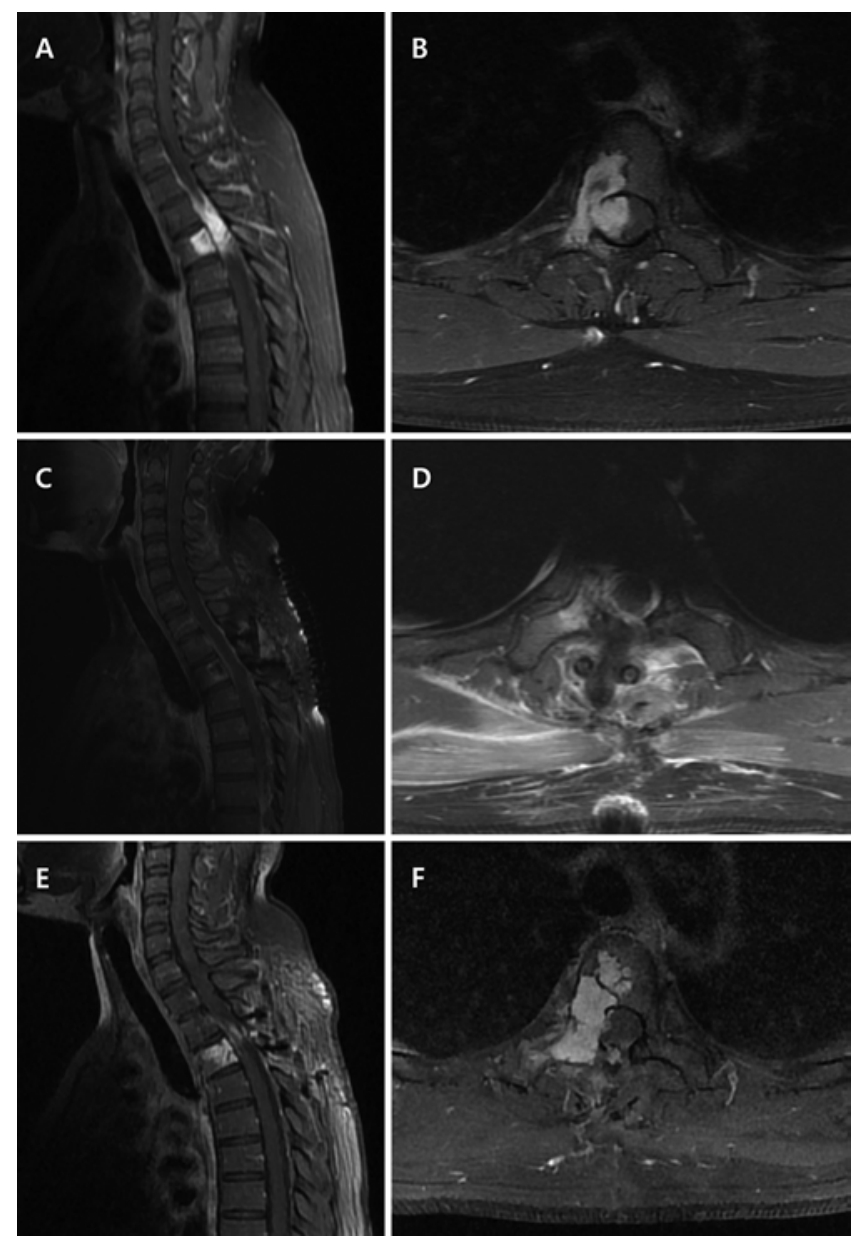

FIG. 1. Sagittal $(A, C$, and $E)$ and axial $(B, D$, and $F) M R$ images of the patient. Preoperative images at the local hospital (A and $B)$ demonstrated an enhancing lesion affecting the right pedicle, lamina, and vertebral body of T3 as well as the epidural space of T2-3. Postoperatively (C and $\mathbf{D})$, the epidural mass was removed but the lesions in the pedicle and vertebral body remained unchanged. Upon presentation to our institute, the mass had increased in size ( $E$ and $F)$.

aortic injury was suspected and the thoracic surgeon was immediately called. At the same time, we started to close the surgical wound rapidly, leaving the temporary pedicle fixation in situ. The thoracic surgeon arrived promptly, but she recommended we contact the cardiac surgeon because it would be a cardiovascular emergency if an aortic injury was strongly suspected.

In the meantime, the anesthesiologist reported a drop in blood pressure and oxygen saturation, even with a massive transfusion. The patient's heart was still beating, but she was turned supine and cardiac massage was initiated to maintain blood pressure. Direct repair of the injured aorta was thought to be the only option to stop the bleeding, and an urgent call was made to the cardiac surgeon. Initially, he recommended endovascular intervention instead of open repair. However, the patient was believed to be too unstable to be transferred to the angiography suite, so we insisted on an open repair. An agreement was reached and the cardiac surgeon soon arrived with his team.

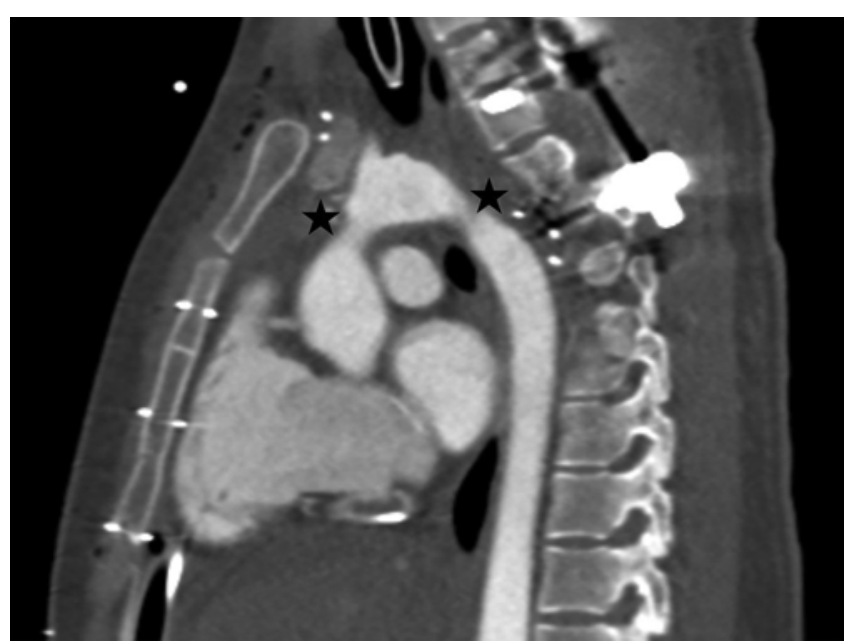

FIG. 2. Postoperative CT image. The sagittal view of the thorax shows the relationship between the spondylectomy site and the aorta. The stars represent the suspected sites of injury.

Given that aortic injury was suspected with ongoing massive bleeding, the cardiac surgeon planned to initiate cardiopulmonary bypass (CPB) to cross-clamp the aorta and repair the aortic injury. While maintaining appropriate mean arterial pressure using intermittent chest compression through monitoring of invasive blood pressure and cerebral oximetry via near-infrared spectroscopy, the femoral artery and vein were punctured by an anesthesiologist with the aid of ultrasonography. A cannula was put into the right femoral artery through the guidewire, but the initial attempt failed. The second attempt was made on the left side and cannulas were successfully inserted into the left side. The CPB was started 1.4 hours after the initial injury.

Sternotomy was performed by the cardiac surgeons, and they found massive arterial bleeding from both the anterior and posterior surface of the aortic arch. During systemic cooling to achieve deep hypothermic circulatory arrest (DHCA), one cardiac surgeon applied manual compression on the bleeding foci while the other surgeon performed a pericardiotomy, superior vena cava (SVC) cannulation for adequate venous drainage, and left ventricle (LV) cannula insertion for LV decompression. After the patient's body temperature reached deep hypothermia, total circulatory arrest was initiated and bleeding foci were explored. An aortotomy procedure at the ascending aorta revealed two bleeding spots: one on the anterior wall of the ascending aorta and the other on the posterior wall of the proximal descending thoracic aorta. It was suspected that the osteotome pierced through the aortic arch during the deepening of the annulus fibrosus incision. The defects on both sides of the aortic arch were successfully repaired under total circulatory arrest and retrograde cerebral perfusion (RCP; Fig. 2).

Even after the repair was complete, a large amount of bleeding was still noted, slowly filling up the thoracic cavity. Also, there was oozing from the posterior surgical wound, as it had been closed roughly. The bleeding was massive, dribbling on the floor of the operating room. The 


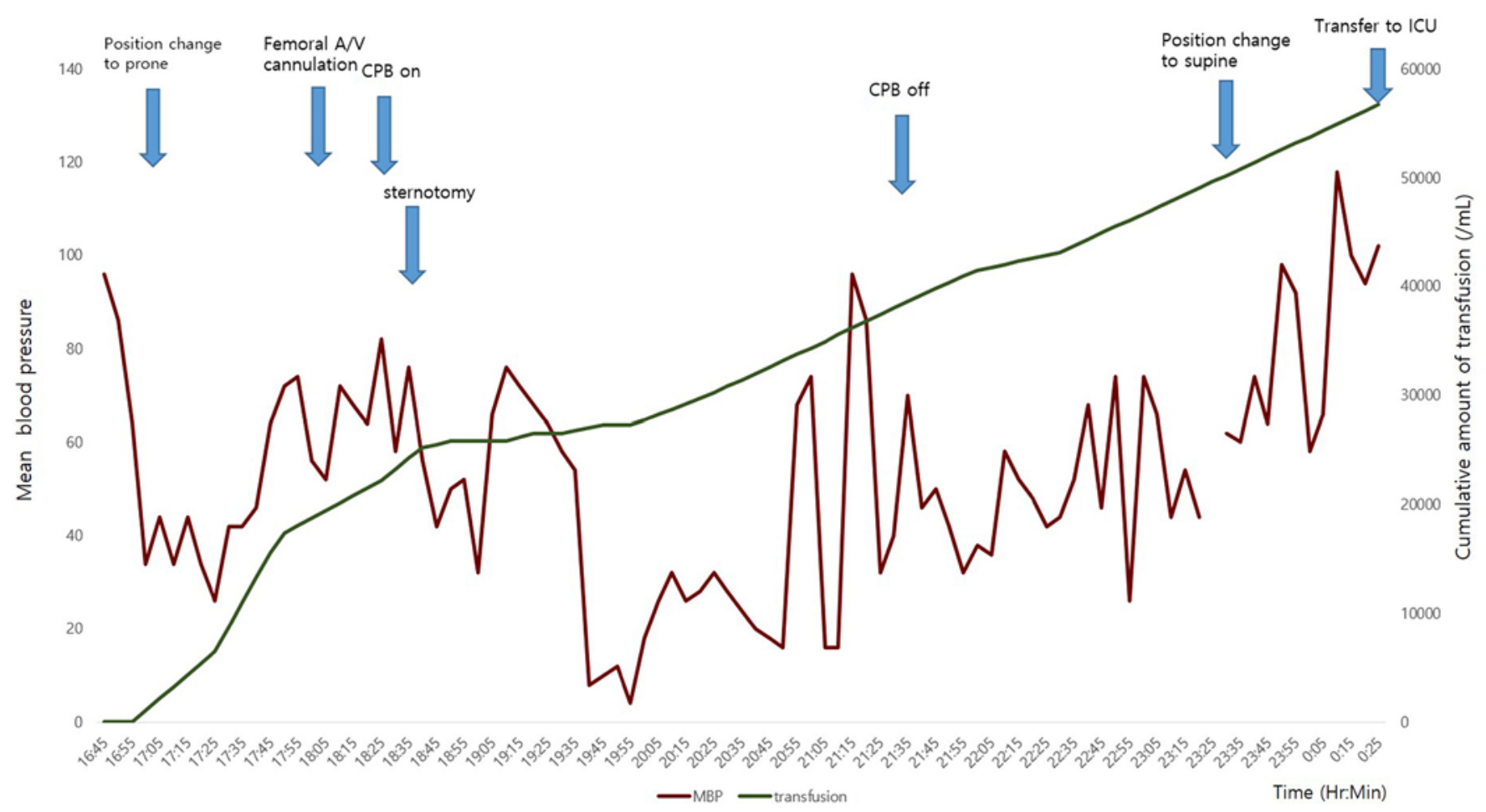

FIG. 3. A graph showing the mean arterial blood pressure of the patient throughout the surgery in relation to major operative events. The green line represents the total amount of blood transfusion during the surgery. A/V = artery/vein; ICU = intensive care unit; $\mathrm{MBP}=$ mean blood pressure. Figure is available in color online only.

cardiac surgeon tried to control it but was unsuccessful. Instead, gauze packing was performed but the bleeding continued, so we decided to revise the posterior wound. After the temporary closure of the sternotomy wound, we turned the patient prone again. As the posterior wound was reopened, the engorged epidural venous plexus was pumping out blood, resembling arteries. It was successfully controlled with bipolar cautery.

After 16 hours of surgery with a massive blood transfusion of $52,715 \mathrm{ml}$, the patient was transferred to the surgical intensive care unit. Immediate after surgery she remained intubated, but was able to obey commands. On postoperative day 1 , she underwent delayed sternal closure. On postoperative day 2, extubation was conducted. A neurological examination revealed grade I-II weakness on the Medical Research Council scale in both legs, which was worse on the right side. Otherwise, she had no other neurological abnormalities. On postoperative day 7 , she underwent another surgery to remove the T3 vertebral body with the remainder of the tumor and to fix adjacent levels of the thoracic spine with screws and rods. After the third operation, she made a good recovery and was transferred to a general ward. Her lower-limb weakness gradually improved. On postoperative day 12 from the initial surgery, she was able to walk with walking aids. She was transferred to the inpatient rehabilitation unit, where she continued her rehabilitation. After a month of hospitalization, she was able to walk unaided and was discharged from the hospital. Six months later, she has had no neurological deficits.
Pathological examination of the resected T3 vertebra revealed a myxoid tumor, revised from the previous diagnosis of choroid meningioma.

\section{Discussion}

The majority of iatrogenic aortic injuries have been described as occurring on the abdominal aorta when lumbar body fusion was performed. ${ }^{1,6,12}$ In fewer cases, injuries of the thoracic aorta have been reported as complications after the thoracic spinal instrumentation. ${ }^{7,8,13}$ To the best of our knowledge, this is the first case to report an intraoperative injury of the aortic arch where an instrument pierced through the aorta.

Due to its proximity to the vertebrae, the aorta is at risk of injury during spinal surgery. Although the injury rate is very low, the number of incidences is likely to increase with the corresponding increase in surgery for the upper thoracic spine in the aging population, with advances in surgical techniques. ${ }^{5}$ Aortic injury is a life-threatening situation if not properly managed. If an aortic injury is suspected, immediate help from a multidisciplinary team must be sought. Timely decisions should then be made regarding the management of patients in the coordination of limited resources. In our case, prompt help from anesthesiologists and cardiac surgeons was available. CPB with therapeutic hypothermia were performed along with RCP via the SVC, with cooling of the patient's head in an attempt to protect the brain.

The CPB is essential in most cases of open-heart sur- 
TABLE 1. Steps taken in the case and procedures that could have been performed to prevent the aortic arch injury

\begin{tabular}{lcc}
\hline \multicolumn{1}{c}{ Operative Procedure } & Steps Taken in the Case & Procedures That Could Have Been Performed to Prevent the Injury \\
\hline Release of ALL & Thoracoscopic release & $\begin{array}{c}\text { Anterior approach, leaving rubber or double-folded Gelfoam } \\
\text { between the vessels and the spine for protection and to serve } \\
\text { as a limit for the posterior approach }\end{array}$ \\
$\begin{array}{l}\text { Posterior release of remaining ligament } \\
\text { and intervertebral disc }\end{array}$ & $\begin{array}{ll}\text { Use of an osteotome in a blind manner } \\
\text { Use of a Kerrison punch under direct vision }\end{array}$ \\
\hline
\end{tabular}

gery, and arterial/venous cannulation is the first step to initiate CPB. ${ }^{10}$ Various cannulation strategies have been established, especially in aortic surgery, in which modification of cannulation according to the lesion to be operated on is mandatory to maintain end-organ perfusion. ${ }^{2}$ In addition, not only cannulation but also the degree of hypothermia (mild vs moderate vs deep hypothermia) is an important component of the CPB strategy.,11 In terms of cannulation, in our case, central cannulation after sternotomy was impossible because of ongoing chest compression; thus, peripheral cannulation via femoral vessels was initially attempted and CPB was started via the femoral vessels. In terms of hypothermia, DHCA (i.e., no perfusion to the entire body including the brain) under $18^{\circ} \mathrm{C}$ is required for exploration of an aortic arch injury and repair under clear direct vision with a bloodless field. In this case, the body temperature, measured by a nasopharyngeal probe, dropped to $16.3^{\circ} \mathrm{C}$. The RCP via the SVC was performed adjunctively to DHCA for the purpose of partial cerebral perfusion and pushing out emboli from the carotid arteries.

Along with the DHCA and RCP, a massive blood transfusion was given instead of crystalloids (a total volume of $52,715 \mathrm{ml}$; 66 units of red blood cells, 62 units of fresh frozen plasma, and 12 units of plateletpheresis) to maintain the effective circulatory volume (Fig. 3). Fortunately, the patient experienced no major transfusion-related complications such as electrolyte imbalance, metabolic acidosis, disseminated intravascular coagulation, transfusion-related acute lung injury, and acute kidney injury. Postoperatively, she had gained $14.6 \mathrm{~kg}$, but her weight returned to the preoperative value on postoperative day 14 .

We believed that effective blood volume reached the brain throughout the surgery with the efforts mentioned above, leaving the patient with no neurological sequelae. She experienced severe weakness postoperatively in her lower limbs, but this progressively improved. Upon discharge, she was able to walk without walking aids. Six months later, she had no neurological deficits. The mechanism responsible for the initial weakness is unclear, but it was thought to be due to ischemia on the thoracic spinal cord, which recovered before progression to spinal cord infarction.

Following this event, we changed our method of performing a T3 spondylectomy. Instead of using a sharp osteotome in a blinded way, we began to use a less-sharp Kerrison punch to remove the annulus fibrosus under direct vision in the posterior-only approach. We believe the ALL may work as a limit for the posterior approach, protecting the great vessels; thus, we did not ask a thoracic surgeon to perform anterior release of the ALL using a thoracoscope. If an anterior approach to the ALL is necessary, rubber or double-folded Gelfoam can be left for protection between the vessels and the spine after anterior release of the ALL; these can serve as a limit for the posterior approach as well (Table 1).

\section{References}

1. Aichmair A, Fantini GA, Garvin S, Beckman J, Girardi FP: Aortic perforation during lateral lumbar interbody fusion. J Spinal Disord Tech 28:71-75, 2015

2. Chalegre ST, Sá MP, de Rueda FG, Salerno PR, Vasconcelos FP, Lima RC: Central versus peripheral arterial cannulation and neurological outcomes after thoracic aortic surgery: meta-analysis and meta-regression of 4459 patients. Perfusion 30:383-388, 2015

3. Engelman R, Baker RA, Likosky DS, Grigore A, Dickinson TA, Shore-Lesserson L, et al: The Society of Thoracic Surgeons, The Society of Cardiovascular Anesthesiologists, and The American Society of ExtraCorporeal Technology: Clinical Practice Guidelines for Cardiopulmonary BypassTemperature Management during Cardiopulmonary Bypass. J Extra Corpor Technol 47:145-154, 2015

4. Fantini GA, Pappou IP, Girardi FP, Sandhu HS, Cammisa FP Jr: Major vascular injury during anterior lumbar spinal surgery: incidence, risk factors, and management. Spine (Phila Pa 1976) 32:2751-2758, 2007

5. Kobayashi K, Imagama S, Ando K, Ishiguro N, Yamashita M, Eguchi Y, et al: Complications associated with spine surgery in patients aged 80 years or older: Japan Association of Spine Surgeons with Ambition (JASA) Multicenter Study. Global Spine J 7:636-641, 2017

6. Kueper J, Fantini GA, Walker BR, Aichmair A, Hughes AP: Incidence of vascular complications during lateral lumbar interbody fusion: an examination of the mini-open access technique. Eur Spine J 24:800-809, 2015

7. Martin S, Lindsay R, Baker RC: Simultaneous endovascular repair of a thoracic aortic injury during posterior pedicle screw removal: a case report. Ann Vasc Surg 48:252. e1-252.e4, 2018

8. Martín-Pedrosa JM, Gutiérrez V, González-Fajardo JA, Vaquero C: Endovascular treatment of thoracic aorta injury after spinal column surgery. J Vasc Surg 55:1782-1783, 2012

9. Oskouian RJ Jr, Johnson JP: Vascular complications in anterior thoracolumbar spinal reconstruction. J Neurosurg 96 (1 Suppl):1-5, 2002

10. Safi HJ, Miller CC III, Lee TY, Estrera AL: Repair of ascending and transverse aortic arch. J Thorac Cardiovasc Surg 142:630-633, 2011

11. Shaaban Ali M, Harmer M, Kirkham F: Cardiopulmonary bypass temperature and brain function. Anaesthesia 60:365-372, 2005

12. Umeda A, Saeki N, Matsumoto C, Nakao M, Kawamoto M: 
Abdominal aortic injury during vertebroplasty. Spine (Phila Pa 1976) 40:E439-E441, 2015

13. Yelamarthy PKK, Mahajan R, Rustagi T, Tandon V, Sangondimath G, Chhabra HS: Aortic rupture during surgical management of tubercular spondylodiscitis. Cureus 10:e2255, 2018

\section{Disclosures}

The authors report no conflict of interest concerning the materials or methods used in this study or the findings specified in this paper.

\section{Author Contributions}

Conception and design: Chung. Acquisition of data: HS Kim. Analysis and interpretation of data: HS Kim. Drafting the article: HS Kim, DH Kim. Critically revising the article: Chung, KH Kim, Cho. Reviewed submitted version of manuscript: Chung, HS Kim, DH Kim. Administrative/technical/material support: DH Kim, KH Kim, Cho. Study supervision: Chung, DH Kim, KH Kim, Cho.

\section{Correspondence}

Chun Kee Chung: Seoul National University Hospital, Seoul, South Korea.chungc@snu.ac.kr. 